\title{
Computer simulation of the pulsating blood flow in arteries with stenosis, aneurysms and plaques
}

\author{
Vladimir Tregubov \\ Saint-Petersburg State University, Russia \\ E-mail:v.tregubov@spbu.ru \\ (Received 19 February 2016; accepted 8 April 2016)
}

\begin{abstract}
The stenosis, aneurysms and plaques are the most common types of the blood vessel pathology. To study their influence on the pulsating blood flow and the internal pressure the mechanical models of pulsating blood flow and the above mentioned pathology of blood vessels were developed. The blood was considered as non-Newtonian liquid. As the boundary condition on the vessel wall the semi-slip regime was chosen. Computer simulation was executed using Finite element method, which was realized by means of the system ABAQUS. As results the pressure and velocity distributions were obtained for the four kinds of pathology in each time moment of the pulse cycle.
\end{abstract}

Keywords: computer simulation, non-Newtonian liquid, plaque, stenosis, aneurism.

\section{Introduction}

Stenosis of blood vessels is the narrowing of their lumen, which causes poor circulation and tissue nutrition. This leads to the hypertrophy of left ventricle and the heart attack and stroke.

An aneurysm is a dilatation of the blood vessel to an area with thinning and weakening of its walls. This extension may be saccular or fusiform shape. An aneurysm is dangerous because the vessel in this place can break at any moment, causing massive bleeding, or haemorrhage. In addition, an aneurysm is a convenient place for the formation of blood clots.

Cholesterol plaques are formed on the rough walls of blood vessels as layers of fat that appears as a result of metabolic disorders. Thus there is an overgrowth of muscle cells and connective tissue that leads to reduced blood flow. This disease can affect arteries of large and medium size. In the area of plaque there is a possibility of the protective endothelium damage, which contributes to the formation of parietal thrombi and the further reduction of blood flow. In the future, these thrombi are often destroyed, and their particles are carried to the small blood vessels, causing the embolism, apoplectic stroke or heart attack.

The majority of authors model the blood by non-Newtonian fluid [1]. This allows, at least partially, to take into account the complexity of the internal blood structure, containing a large number of blood cells. The modelling of pulsating flow of blood as non-Newtonian liquid for deformable arteries without pathologies was presented in [2]. The mathematical model of atherosclerosis plaque formation was proposed in [3], where the blood was considered as an incompressible non-Newtonian fluid with shear-thinning viscosity. The wall shear stress and blood velocity behaviour in a vessel with two plaques in different positions were obtained. The numerical simulation of Non-Newtonian blood flow in a stenosis vessel was presented in [4]. The vessel was considered as two-dimensional channel and plaque area is modelled as a homogenous porous medium. The non-Newtonian micropolar fluid was used in [5] for mathematical modelling of the arterial blood flow through a composite stenosis.

But even in all these cases, pointed above, the blood can be considered as a liquid only in large and medium arteries. A number of researches attempt to model the blood as two-phase liquid. Thus, in [6] both phases are liquids with different properties. The two-phase non-linear model was used in [7] for the modelling of flow through the stenosed blood vessels. The most difficulty of 
this approach consists in that mechanical properties of blood cells are not clearly understood as yet.

\section{Mechanical model and computer simulation}

Four kinds of pathology were considered in the proposed model: saccular and fusiform aneurysms, stenosis and plaque. They were modelled by means of non-deformable cylinders with a diameter $2 \mathrm{~cm}$ and spheres with smooth connections, as it is shown in Fig. 1. The blood was considered as non-Newtonian liquid with the power law dependence of the stress tensor $\mathbf{T}$ from the strain velocity tensor $\mathbf{D}$. The governing equations in this case can be written as follows:

$$
\left\{\begin{array}{l}
\rho \frac{d \mathbf{v}}{d t}=\nabla \cdot(-p \mathbf{I}+\mathbf{T}) \\
\operatorname{div} \mathbf{v}=0 \\
\mathbf{T}=2 k\left|I_{2}\right|^{\left(\frac{n-1}{2}\right)} \mathbf{D}
\end{array}\right.
$$

where $\mathbf{v}$ is the velocity vector, $\rho$ is the density, $p$ is the pressure, $\mathbf{I}$ is the unit vector, $I_{2}$ is the second invariant, $k$ and $n$ are the coefficients, which define the specific kind of power law. The pulsating velocity profile was set at the entrance cross-section of the considered artery segment.

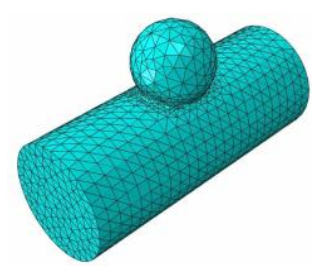

a

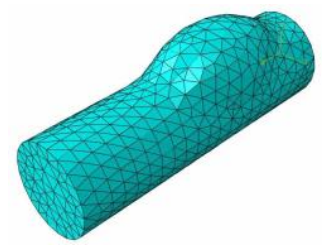

b

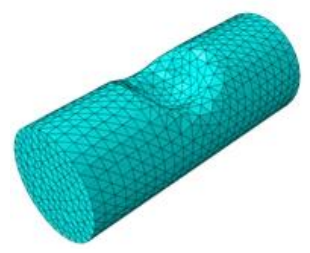

c

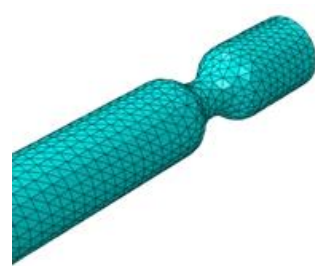

d

Fig. 1. The mechanical models of a) saccular aneurysm, b) fusiform aneurysm, c) plaque, d) stenosis

In distinction to the papers cited above the so called "semi-slip" condition was used as a boundary condition at the vessel wall. This allows us to take into account the specificity of the interaction of the blood cells and plasma with the vessel wall. It can be written as follows:

$\left.v_{\tau}\right|_{\text {wall }}=-\left.b\left(\frac{\partial v_{\tau}}{\partial n}\right)\right|_{\text {wall }}$,

where $v_{\tau}$ is the tangent velocity, $n$ in the normal to the vessel inner surface, $b$ is the coefficient.

Computer simulation was executed using Finite element method, which was realized by means of the system ABAQUS, which has high-powered means for the creation of a model geometry and visualization of the results. For this purpose, the meshes of the first order were used, as it is shown in Fig. 1.

\section{Results}

As results the pressure and velocity distributions were obtained for the four kinds of pathology in each time moment of the pulse cycle. The examples of the pressure distribution are shown in Fig. 2 and of the velocity distribution in Fig. 3. It is also possible to construct the velocity profiles in any cross-section. 


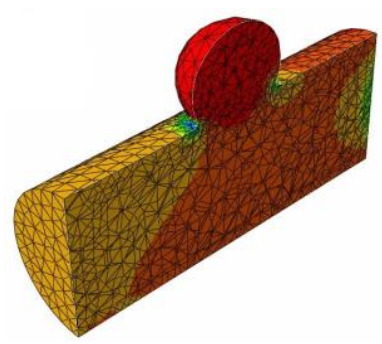

a

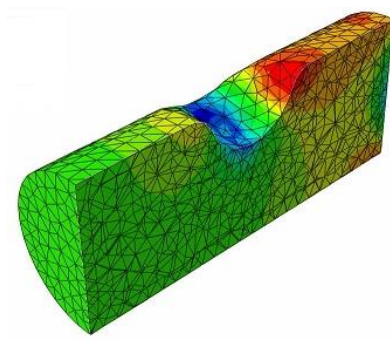

$\mathrm{b}$

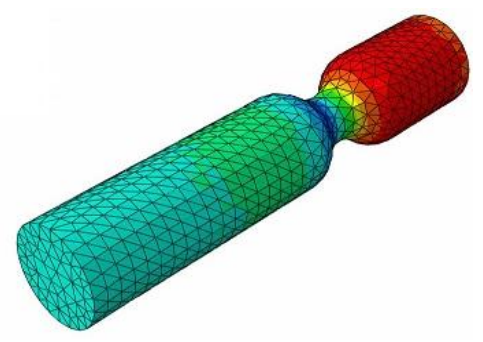

c

Fig. 2. The pressure distribution in time moment $0.5 \mathrm{sec}$ in a) saccular aneurism, b) plaque, c) stenosis

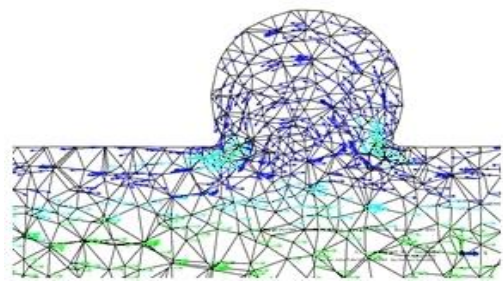

a

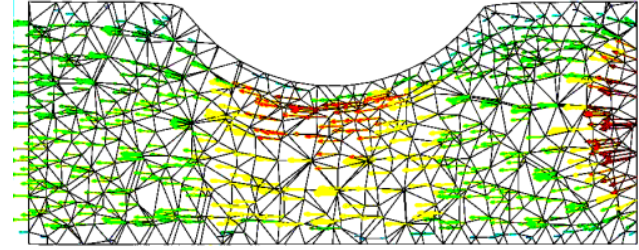

$\mathrm{b}$

Fig. 3. The velocity distribution in in time moment $1 \mathrm{sec}$ in a) saccular aneurism, b) plaque

\section{Conclusion}

The information, received by means of computer simulation, make it possible to demonstrate how the pulse blood flow and pressure are changed in the presence of certain types of vascular pathology. In particular, the origin and disappearance of vortexes, the return wall-adjacent blood flow and the appearance of the critical pressure areas were observed. The obtained results allow also us to construct the velocity profiles in each cross-section.

\section{References}

[1] Dutta, A.; Tarbell, J. M. 1996. Influence of non-Newtonian behavior of blood on flow in an elastic artery model, Journal of Biomechanical Engineering 118(1): 111-119.

http://dx.doi.org/10.1115/1.2795936

[2] Ratkina, S. V.; Tregubov, V. P. 2010. Mathematical simulation of a blood stream in large arteries, in Biomechanics 2010: International Conference of Polish Society of Biomechanics. Book of abstracts, August 25-28, 2010, Warsaw, Poland. Warsaw: Warsaw University of Technology, 187-188.

[3] Silva, T., et al. 2013. Mathematical modeling of atherosclerotic plaque formation coupled with a nonNewtonian model of blood flow, Conference Papers in Mathematics 2013: 405914.

http://dx.doi.org/10.1155/2013/405914

[4] Shojaeizadeh, M.; Yeganegi, A. R. 2015. Effects of the non-Newtonian viscosity of blood on flow field in a constricted artery with a porous plaque. International Journal of Science and Engineering Investigations 4(38): 15-18.

[5] Ellahi, R., et al. 2014. A mathematical study of non-Newtonian micropolar fluid in arterial blood flow through composite stenosis, Applied Mathematics \& Information Sciences 8(4): 1567-1573. http://dx.doi.org/10.12785/amis/080410

[6] Srivastava, V. P. 1996. Two-phase model of blood flow through stenosed tubes in the presence of a peripheral layer: applications, Journal of Biomechanics 29(10): 1377-1382.

http://dx.doi.org/10.1016/0021-9290(96)00037-1

[7] Sankar, D. S.; Lee, U. 2007. Two-phase non-linear model for the flow through stenosed blood vessels, Journal of Mechanical Science and Technology 21(4): 678-689.

http://dx.doi.org/10.1007/BF03026973 